\title{
Laser-ablation-assisted microparticle acceleration for drug delivery
}

\author{
V. Menezes ${ }^{\mathrm{a})}$ and K. Takayama \\ Interdisciplinary Shock Wave Research Center, TUBERO, Tohoku University, 2-1-1 Katahira, \\ Aoba Ku, Sendai 980-8577, Japan \\ T. Ohki \\ Interdisciplinary Shock Wave Research Center, Institute of Fluid Science, Tohoku University, \\ 2-1-1 Katahira, Aoba Ku, Sendai 980-8577, Japan \\ J. Gopalan \\ Aerospace Engineering Department, Indian Institute of Science, Bangalore 560-012, India
}

(Received 18 May 2005; accepted 22 August 2005; published online 12 October 2005)

Localized drug delivery with minimal tissue damage is desired in some of the clinical procedures such as gene therapy, treatment of cancer cells, treatment of thrombosis, etc. We present an effective method for delivering drug-coated microparticles using laser ablation on a thin metal foil containing particles. A thin metal foil, with a deposition of a layer of microparticles is subjected to laser ablation on its backface such that a shock wave propagates through the foil. Due to shock wave loading, the surface of the foil containing microparticles is accelerated to very high speeds, ejecting the deposited particles at hypersonic speeds. The ejected particles have sufficient momentum to penetrate soft body tissues, and the penetration depth observed is sufficient for most of the pharmacological treatments. We have tried delivering $1 \mu \mathrm{m}$ tungsten particles into gelatin models that represent soft tissues, and liver tissues of an experimental rat. Sufficient penetration depths have been observed in these experiments with minimum target damage. (C) 2005 American Institute of Physics. [DOI: 10.1063/1.2093930]

Localized, needle-free drug delivery is emerging as an effective technique to transfer adequate concentrations of pharmacologic agents directly to delicate and nonapproachable treatment sites in the body with minimum side effects. Moreover, the direct use of drugs in powder form can be very useful in the treatment of certain cancer cells, thrombosis, and in gene therapy. In recent years, several devices have been configured and tested for delivering microparticles at controlled velocities into soft targets. Klein et al. ${ }^{1}$ used a detonation driven particle gun to deliver drug adsorbed microtungsten particles into plant cells. A needle-free vaccine delivery system is successfully developed and tested, in which pressurized helium gas in conjunction with a miniature nozzle has been used to accelerate powdered pharmaceutical agents to enter the epidermis of human skin. ${ }^{2-4}$ Emergence of these localized drug delivery techniques have opened up new vistas in gene therapy and pharmacological treatments.

Among the drug delivery methods mentioned earlier, the detonation driven particle gun ${ }^{1}$ is a nonintrusive technique, which delivers dry particles into the target. Though it can prove to be a potential technique in delivering drugs into plant cells, it may not be suitable for clinical/surgical procedures since an explosive charge is used for detonation, and also miniaturization of such a device may not be possible. The needle free vaccine delivery system ${ }^{2-4}$ offers the potential to deliver vaccines into layers of skin in a controlled manner to achieve a pharmacological effect, and is a compact hand held device that can be self-administered by the patients. But, since a substantial quantity of helium gas (carrier gas) flows along with the particles in this device, it may not find applications in delivering vaccines to internal treat-

\footnotetext{
${ }^{a)}$ Electronic mail: viren@rainbow.ifs.tohoku.ac.jp
}

ment sites in the body. Moreover, design of this device is such that the outlet of the miniature nozzle has to be in firm contact with the target to achieve successful particle penetration, which makes the device intrusive for surgical procedures.

The developments explained earlier are quite amazing, but still we do not have a practical particle delivery device that can be easily integrated with medical surgical procedures to deliver drug coated dry particles into soft internal body targets. Under the Center of Excellence program of the Japanese Ministry of Science and Education, we at the Interdisciplinary Shock Wave Research Center of Tohoku University initiated the development of a laser ablation based microparticle delivery device that can be integrated with endoscopic surgical techniques. Lasers are widely used in surgical procedures since their operation can be precisely controlled. Hence, though a laser ablation is used to accelerate the particles, owing to its controllability and nanopulse duration, it is safe for medical use. In this letter, we report the particle delivery device, the involved flow physics and some of the sample results on particle delivery into soft targets.

The device consists of a $Q$-switched Nd: Yttriumaluminum-garnet laser (1064 $\mathrm{nm}$ wavelength, $1.4 \mathrm{~J} /$ pulse energy, $5.5 \mathrm{~ns}$ pulse duration), the optical setup, and a $100-\mu \mathrm{m}$-thick aluminum foil with a 5 -mm-thick BK7 glass overlay as diagramed in Fig. 1. A thin layer of microparticles that are to be accelerated is deposited on one of the surfaces of the foil and the second (back) surface is irradiated with the laser beam with an energy of $0.25 \mathrm{GW}$. The deposited laser energy causes vaporization of a small portion of the foil. The expanding ionized foil vapor drives a shock wave through the foil, which reflects as an expansion wave from the end of the foil surface due to the acoustic impedance mismatch be- 

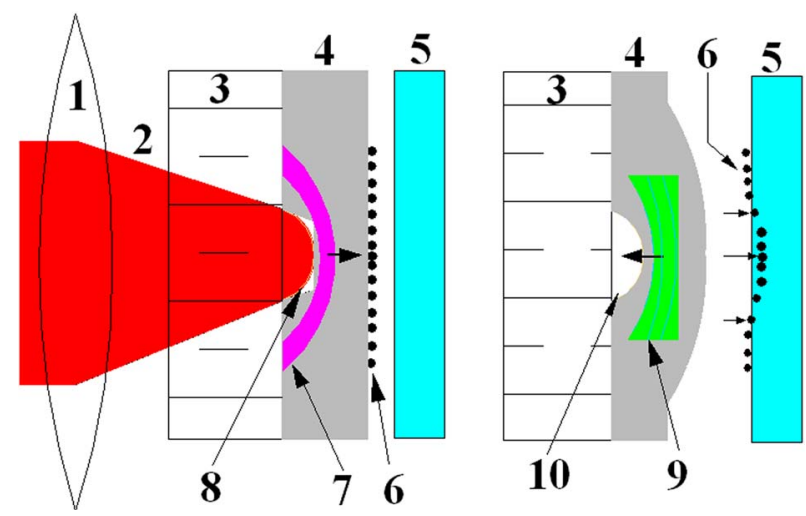

FIG. 1. (Color online) Schematic of laser ablation and foil/particle acceleration. [1 Lens. 2 Laser beam. 3 Glass overlay. 4 Foil. 5 Target. 6 Particles. 7 Shock wave. 8 Confined ablation. 9 Expansion wave. 10 Microcrater due to ablation.]

tween the foil and the air. The backward propagation of the expansion wave would cause the foil to deform suddenly in the opposite direction, ejecting the deposited layer of particles at a very high speed. The BK7 glass overlay helps confining the laser ablation, making the shock wave stronger.

Based on the plastic deformation in the foil due to shock wave loading, the surface velocity of the foil can be estimated from the first principles using basic continuum mechanics. ${ }^{5}$ The shock wave propagating through the foil is a longitudinal compressive wave, and its velocity $C_{l}$ in a thin metal foil can be given by

$$
C_{l}=\sqrt{\frac{E(1-\nu)}{\rho(1+\nu)(1-2 \nu)}},
$$

where $E$ is the Young's modulus, $\rho$ is the density, and $\nu$ is the Poisson's ratio of the material in which the compressive wave is propagating. For an aluminum foil, these values are (E) $70 \mathrm{GPa},(\rho) 2700 \mathrm{~kg} / \mathrm{m}^{3}$, and $(\nu) 0.33$, respectively. So the velocity of the compressive wave in aluminum foil works out to be $\left(C_{l}\right) 6198 \mathrm{~m} / \mathrm{s}$.

If the compressive/shock wave causes a step increase in pressure $(P)$ in the foil, then the displacement $(S)$ of the foil due to shock wave loading can be given by

$$
S=\frac{2 P C_{l} \tau}{E}
$$

where $\tau$ is the time needed for the wave to travel once through the thin foil. The displacement $(S)$ of the foil is the plastic deformation in the foil due to shock wave loading, and can physically be measured. Knowing the value of $C_{l}$, the value of $\tau$ can be calculated for a $100-\mu \mathrm{m}$-thick aluminum foil. The measured plastic deformation in the foil for a confined laser ablation with an energy deposition of $0.25 \mathrm{GW}$ is about $185 \mu \mathrm{m}$, and with this, the pressure $(P)$ induced in the foil is estimated to be about $65.3 \mathrm{GPa}$ from

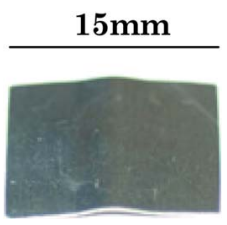

FIG. 2. (Color online) 100- $\mu \mathrm{m}$-thick aluminum foil deformed due to shock

wave loading.
Downloaded 09 Dec 2005 to 203.200 .43 .195$. Redistribution subject to AlP license or copyright, see http://apl.aip.org/apl/copyright.jsp

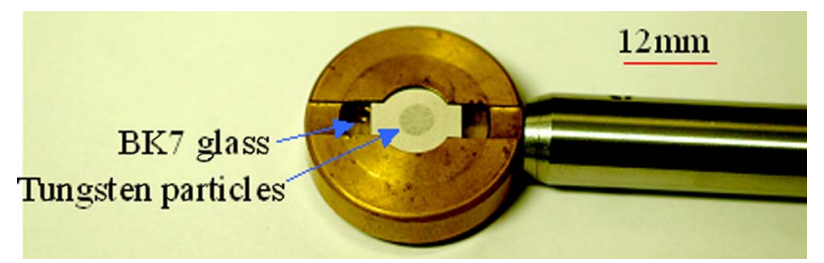

FIG. 3. (Color online) A thin layer of $1 \mu \mathrm{m}$ tungsten particles deposited on an aluminum foil.

Eq. (2). On knowing the values of $P$ and $C_{l}$, the velocity $(V)$ of the foil can be calculated from the following equation:

$$
V=\frac{P C_{l}}{E},
$$

where the value of $V$ works out to be $5782 \mathrm{~m} / \mathrm{s}$. Since the foil thickness is very small, the surface of the foil on which the particles are deposited is expected to accelerate to this velocity and so are the particles. Hence, the deposited particles will initially accelerate to about $5782 \mathrm{~m} / \mathrm{s}$ with a confined energy deposition of $0.25 \mathrm{GW}$. The deformed 100 - $\mu \mathrm{m}$-thick aluminum foil after shock wave loading is shown in Fig. 2.

The particles to be deposited are suspended in a solvent $\left(\mathrm{C}_{2} \mathrm{H}_{5} \mathrm{OH}\right.$ or 2-propanol) and a small volume (typically $2.5-5 \mu \mathrm{L}$ ) of this suspension is deposited on the thin foil. The solvent evaporates leaving behind a thin trace of suspended particles. Depending upon the desired distribution and number of particles, the concentration (ppm level) of the particles and the volume to be deposited are varied. A thin layer of $1 \mu \mathrm{m}$ tungsten particles deposited on a $100-\mu \mathrm{m}$-thick aluminum foil is shown in Fig. 3.

$1 \mu \mathrm{m}$ tungsten particles are delivered into soft targets such as 3\% gelatin and liver tissue of an experimental rat (Sprague Dawley male). The 3\% gelatin (20-25 bloom, cooled at $10{ }^{\circ} \mathrm{C}$ for $1 \mathrm{~h}$ ) represents a human thrombus model and a penetration of about $1 \mathrm{~mm}$ has been observed in this case. The percentage of gelatin is determined by weight ratio of gelatin to water. A gelatin model with the penetrated tungsten particles is shown in Fig. 4. Figure 5 shows $1 \mu \mathrm{m}$ tungsten particles delivered into liver tissues of Sprague Dawley male rat. The sections shown are hematoxylin-eosin stained and are 30-50 $\mu \mathrm{m}$ thick. Most of the penetration is observed along the focal point of the laser beam. Four experimental animals (rats) have been used for this study so far, and a good repeatability has been observed in the results.

Knowing the initial velocity of the particles from Eq. (3), the velocity of the particles at or near the surface of the target can be deduced from the equations of motion, assuming an appropriate size for the particles. If we assume a particle sphere of about $3 \mu \mathrm{m}$ diameter (in the case of cluster-

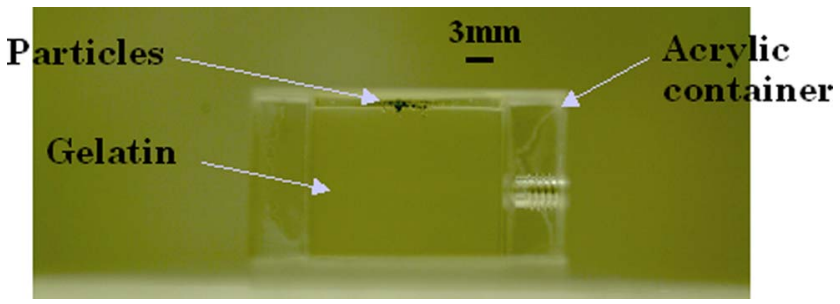

FIG. 4. (Color online) A 3\% gelatin model with penetrated tungsten particles; target standoff distance: $10 \mathrm{~mm}$.

AIP license or copyright, see http://apl.aip.org/apl/copyright.jsp 


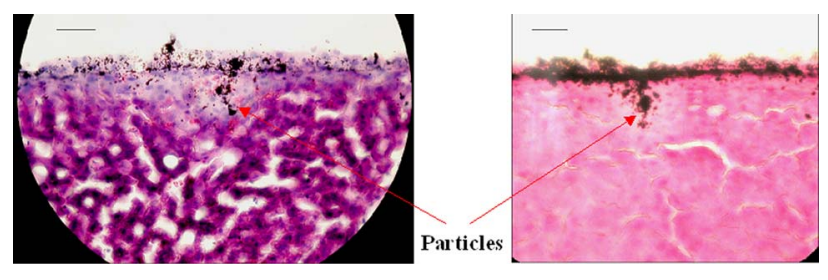

FIG. 5. (Color online) Tungsten particles delivered into Rat liver tissues: (a) sample 1; (b) sample 2. Scale bar: $50 \mu \mathrm{m}$.

ing), flying at $5700 \mathrm{~m} / \mathrm{s}\left(V_{\infty}\right)$ in atmospheric air (density, $\left.\rho_{\infty} \approx 1.18 \mathrm{~kg} / \mathrm{m}^{3}\right)$, its coefficient of $\operatorname{drag}\left(C_{d}\right)$ can be given by the following equation:

$$
C_{d}=\frac{D}{0.5 \rho_{\infty} V_{\infty}^{2} A}
$$

where $D$ is the drag force and $A$ is the frontal surface area of the sphere. At hypersonic speeds, the $C_{d}$ for a sphere can be assumed to be unity. With this, from Eq. (4), the drag force on the particle can be calculated. The equation of motion for such a particle can be given as later

$$
-D+w=m \frac{d V}{d t}
$$

where $w$ is the weight, $m$ is the mass and $d V / d t$ is the deceleration of the particle. Weight of the particle in this case is negligible and, hence, knowing the density of the particle, the deceleration of the particle can be found out. The final velocity of the particle $\left(V_{p}\right)$ can be computed using the following expression:

$$
V_{p}=\left[V_{\infty}^{2}-\left(2 \times \frac{d V}{d t} \times S d\right)\right]^{1 / 2},
$$

where $S d$ (stand-off distance) is the distance of the target from the thin metal foil. In this case, for a particle size of $3 \mu \mathrm{m}$ diameter and target stand-off distance of $10 \mathrm{~mm}, V_{p}$ works out to be about $4700 \mathrm{~m} / \mathrm{s}$.

A theoretical penetration model proposed by $\operatorname{Dehn}^{6}$ has been used to determine the penetration depth of microparticles in rat liver. The force of deceleration acting on each particle is split into an inertial force, required to accelerate the target material up to the speed of the particle, and a static force required to yield the target material ${ }^{4}$

$$
F=\frac{1}{2} \rho_{t} A_{p} V^{2}+3 A \sigma_{y},
$$

where $F$ is the force acting on the particle, $V$ is the instantaneous particle velocity, $A$ is the projected area of the projectile, $\sigma_{y}$ is the yield strength, $\rho$ is the density, and subscripts $p$ and $t$ apply to the particle and target, respectively. Integration of Eq. (7) yields a theoretical penetration depth relation as later

$$
d=\frac{4 \rho_{p} r_{p}}{3 \rho_{t}}\left\{\ln \left[\frac{1}{2} \rho_{t} V_{i}^{2}+3 \sigma_{t}\right]-\ln \left[3 \sigma_{t}\right]\right\} .
$$

In expression (8), $d$ is the penetration depth, $\rho$ is the density, $r$ is the radius of the particle, $V_{i}$ is the particle impact velocity, and $\sigma$ is the yield strength. Subscripts $p$ and $t$ apply to the particle and target, respectively. Assuming that the target is humid (with a lot of water/liquid content), and the liver tissue fails along its cell boundaries considering the particle size (clusters), an yield strength of $1 \mathrm{MPa}$ is assumed for the rat liver. This is a typical value for the yield strength of a humid soft body tissue. ${ }^{7}$ Density for the rat liver is measured to be $1120 \mathrm{~kg} / \mathrm{m}^{3}$. For the tungsten particles used, the density is $19250 \mathrm{~kg} / \mathrm{m}^{3}$ and the particle diameter is assumed to be $3 \mu \mathrm{m}$ (assuming clusters). The impact velocity at the target surface is taken as $4.7 \mathrm{~km} / \mathrm{s}$ as obtained from expression (6). With these values, the theoretical penetration depth of tungsten particles in rat liver tissue works out to be about $287 \mu \mathrm{m}$, which is close to the one shown in Fig. 5 as far as the order of magnitude of the penetration depth is concerned.

Delivery of the microparticles into the rat liver substantiates the usability of the device on internal body organs. The proposed device can also be used to deliver vaccines into human skin. For a successful delivery of vaccines into skin, the drug-coated particles should penetrate the stratum corneum of the skin and reach the viable epidermis. Considering an yield strength and a density of $3.2 \mathrm{MPa}$ (under humid conditions) and $1500 \mathrm{~kg} / \mathrm{m}^{3}$, respectively, for the stratum corneum, ${ }^{8}$ expression (8) gives a penetration depth of about $191 \mu \mathrm{m}$ for the earlier particle size and impact velocity. The estimated penetration depth in skin is 33\% lower when compared to the liver. Based on the experimentally observed penetration depth in liver (Fig. 5), a 33\% lower penetration depth would amount to about $50 \mu \mathrm{m}$, which is sufficient to reach the viable epidermis of the skin, considering a maximum thickness of $16 \mu \mathrm{m}$ for the stratum corneum.

In conclusion, we have demonstrated a laser ablation based particle delivery device to deliver drug coated dry microparticles into soft body targets. Particle penetration depth in the target observed in the preliminary experiments is believed to be sufficient for most of the pharmacological treatments. The device being proposed is supposed to be totally nonintrusive and is likely to have potential applications in medical surgical procedures. Further, it is intended to have a very uniform and controlled distribution of the particles on the foil, such that the particles enter the target as individual drug coated spheres so that a good distribution of drug can be had in the target tissue. It would be very effective if an individual drug coated particle enters a cell in the tissue by rupturing cell membrane, without collapsing the cell boundaries. Also, to apply this device to clinical procedures, it has to be handy and, hence, has to be miniaturized and also has to be integrated with devices like endoscopes. Laser focusing/irradiation in such a case can conveniently be done by drawing an optical fiber from the laser. And if the lenses are miniaturized, then they can easily be integrated with an endoscope, and the device can suitably be used for even endoscopic surgical procedures, when the treatment sites are nonapproachable. These further thoughts would be implemented in the next phase of research.

${ }^{1}$ T. M. Klein, E. D. Wolf, R. Wu, and J. C. Sanford, Nature (London) 327, 70 (1987).

${ }^{2}$ N. J. Quinlan, M. A. F. Kendall, B. J. Bellhouse, and R. W. Ainsworth, Shock Waves 10, 395 (2001).

${ }^{3}$ M. A. F. Kendall, Shock Waves 12, 23 (2002).

${ }^{4}$ M. Kendall, T. Mitchell, and P. Wrighton-Smith, J. Biomech. 37, 1733 (2004).

${ }^{5} \mathrm{~L}$. Brekhovskikh and V. Goncharov, Mechanics of Continua and Wave Dynamics (Springer, Tokyo, 1982).

${ }^{6}$ J. Dehn, Int. J. Impact Eng. 5, 239 (1987).

${ }^{7}$ T. J. Mitchell, M. A. F. Kendall, and B. J. Bellhouse, Int. J. Impact Eng. 28, 581 (2003).

${ }^{8}$ M. Kendall, S. Rishworth, F. Carter, and T. Mitchell, J. Invest. Dermatol. 122, 739 (2004). 